\title{
Effects of Different Suture Materials Used for the Repair of Hypospadias: A Stereological Study in a Rat Model
}

\author{
Mehdi Shirazi Ali Noorafshan Ali Serhan \\ Histomorphometry and Stereology Research Center, Shiraz University of Medical Sciences, Shiraz, Iran
}

\section{Key Words}

Urethra · Suture materials • Urethroplasty • Hypospadias •

Stereology

\begin{abstract}
Introduction: Repair of the penile urethra in hypospadias is done with different suture materials. The aim of this study was to quantify the impact of different suture threads on histological parameters of the penis and urethra following hypospadias repair in rats. Materials and Methods: Six groups of the rats, except for the control group, underwent incision and repair of the penis and urethra in the ventral line using different suture materials including Chromic, Vicryl (polyglactic acid), PDS (polydioxanone), Rapid Vicryl (polyglactin 910), Monocryl (poliglecaprone 25) and control groups. After 3 weeks the body of the penis was excised and histological sections were studied using stereological methods. Results: Monocryl (poliglecaprone 25) was associated with a higher percentage of vessel density, higher volume of urethral lumen and lower lymphocyte infiltration. Volume of urethral epithelium was highest in the PDS group compared to the other operated groups. There was no significant difference regarding collagen deposition and fibroblast infiltration among the operated groups. Conclusions: The Monocryl and PDS groups showed better results.
\end{abstract}

Copyright $\odot 2012$ S. Karger AG, Basel
(C) 2012 S. Karger AG, Basel

0042-1138/12/0894-0395\$38.00/0

Fax +4161306 1234

E-Mail karger@karger.ch

www.karger.com
Accessible online at:

www.karger.com/uin

\section{Introduction}

Urethroplasty or repair of penile urethra in hypospadias is an important subject of urologic surgery [1-3]. In hypospadias surgery the most important issue is preservation of the function and esthetic outcomes [4]. The outcome of hypospadias repair surgery depends on the technique and the instrumentation including suture material used and the suturing technique employed [4-7]. Choosing appropriate suture material for hypospadias surgery depends on several factors including personal preferences, availability and types of material. However, suture material used for urethroplasty should be absorbable and should have enough tensile strength in order to be able to resist the urinary flow after catheter removal [4].

Synthetic absorbable sutures include polyglycolic acid, polyglactic acid, and polydioxanone. They are either monofilament or braided, which can be used in different situations. These sutures are hypoallergenic and do not allow fluids and thereby infection to penetrate the body along the suture tract [8]. Although most urologist surgeons believe that monofilament absorbable sutures are less traumatic and have better outcomes, other studies have revealed that braided sutures have better outcomes [9]. Direction of incision, tensile strength of the skin as well as absorption time of the suture material also affect the outcome of repair. 
There are controversial results in available studies regarding suture materials used in hypospadias repair surgery [9-11]. Some authors believe that using absorbable sutures with a long absorption time (such as polydioxanone) is associated with better outcome [9], although others suggest that polyglycolic acid and polydioxanone sutures should be avoided in hypospadias surgery because of their prolonged resorption and excess reaction [10].

In the present study the penis of rats was incised from the skin to the urethra and sutured with different materials to model the hypospadias. To achieve the goal of this study, in addition to the usual measurement of collagen deposition, the other parameters should be considered quantitatively. Healing is characterized by four distinct, but overlapping phases including hemostasis, inflammation, proliferation and remodeling [12]. The lymphocytes enter the wound area at the later stage of inflammation, and in the present study the numerical density of these cells was considered as a criterion for inflammatory cell accumulation [12]. The major cell of the proliferative phase is the fibroblast. This cell is responsible for initiating angiogenesis, epithelialization, and collagen formation. Thus the numerical density of the fibroblasts should also be estimated. It is remarkable to survey the epithelialization process of the urethral lumen. To identify the effects of different suture materials, the volume (total amount) of the urethral epithelium was the other considered criteria in the present study. Local factors in the wound microenvironment such as low $\mathrm{pH}$, reduced oxygen tension and increased lactate actually initiate vascularization [12]. It is worth considering to check if there are any effects of different suture materials on the process of vascularization. Therefore, the other considered parameter was vessel density in the incised area of the penis.

Therefore, the present study aimed to evaluate some structural changes of the penis, urethra, and fibroblast and lymphocyte numerical density, following urethroplasty with different suture threads - composed of different materials - using stereological methods. The methods provide accurate and quantitative data to compare the changes in different groups.

Up to now, different absorbable suture materials are used for hypospadias repair worldwide. Although some prefer polydioxanone (PDS) because of its lower stricture rate [12], others prefer chromic sutures because of their better cosmetic outcome and less stricture rate [13]. It has also been demonstrated that Vicryl sutures are associated with higher rates of urethrocutaneous fistulas [7]. Despite these scattered studies, data regarding the effects of different absorbable suture materials (Chromic, Monocryl, PDS, Rapid Vicryl and Vicryl) on different stages of the wound-healing process in hypospadias repair is scarce. Thus we performed this experimental stereologic study in order to identify the suture material that creates minimal fistula, less stricture rates and maximum collagen deposition following hypospadias repair in a rat model.

\section{Materials and Methods}

\section{Animals}

Thirty Sprague-Dawley male rats weighting between 280 and $320 \mathrm{~g}$ were included in this study. All rats were housed individually and fed standard food throughout the experiment. One animal was housed per cage with water and food ad libitum. The study was approved by the Research and Ethics Committee of Shiraz University of Medical Sciences. The animals were treated according to the guidelines for the care and handling of laboratory animals provided by Shiraz Laboratory Animals Center in accordance with global standards for laboratory guidelines. These 30 rats were randomly allocated to six equal groups ( 5 in each group). According to standard stereological articles, 5 animals are sufficient in each group [13]. The groups included Chromic (chromic catgut), Vicryl (polyglactic acid), PDS (polydioxanone), Rapid Vicryl (polyglactin 910), Monocryl (poliglecaprone 25), and control groups [11].

\section{Urethral Incision and Repair}

All animals, except those in the control group, were sedated using intramuscular ketamine $(10-15 \mathrm{mg} / \mathrm{kg})$. The external genital area was well shaved and prepared with a povidone-iodine topical antiseptic solution, and then draped with sterile sheets. Under aseptic conditions, the penis was exposed and a French catheter (gauge 3) was inserted into the urethra. After catheterization, an incision was made on the ventral skin of the penis from base to glans, over the midraphe line, continuing deep to open the dartos muscle and the corpus spongiosum. The urethra was visualized and further excised over the mentioned catheter.

Urethroplasty was performed after incising the urethra. The layers were closed sequentially from the urethra to skin by a different suture material $6 / 0$ (chromic catgut, polyglactic acid, polydioxanone, polyglactin 910 , poliglecaprone 25) for each group. The urethra was the first layer to be sutured over the catheter by running suture (6/0) until completely closed. The corpus spongiosum, dartos and skin were also closed using the same suture material. After closure, the catheter was removed, and no dressing was applied, but an oxytetracycline spray was used instead. The rats included in control group were kept in laboratory standard conditions without undergoing any operation or urethral repair.

After operating the groups with five different suture materials, the rats were observed daily for 3 weeks and for bleeding, hematoma formation or other complications. Three weeks after the surgery all penises were cut and deposited in buffered formaldehyde. 


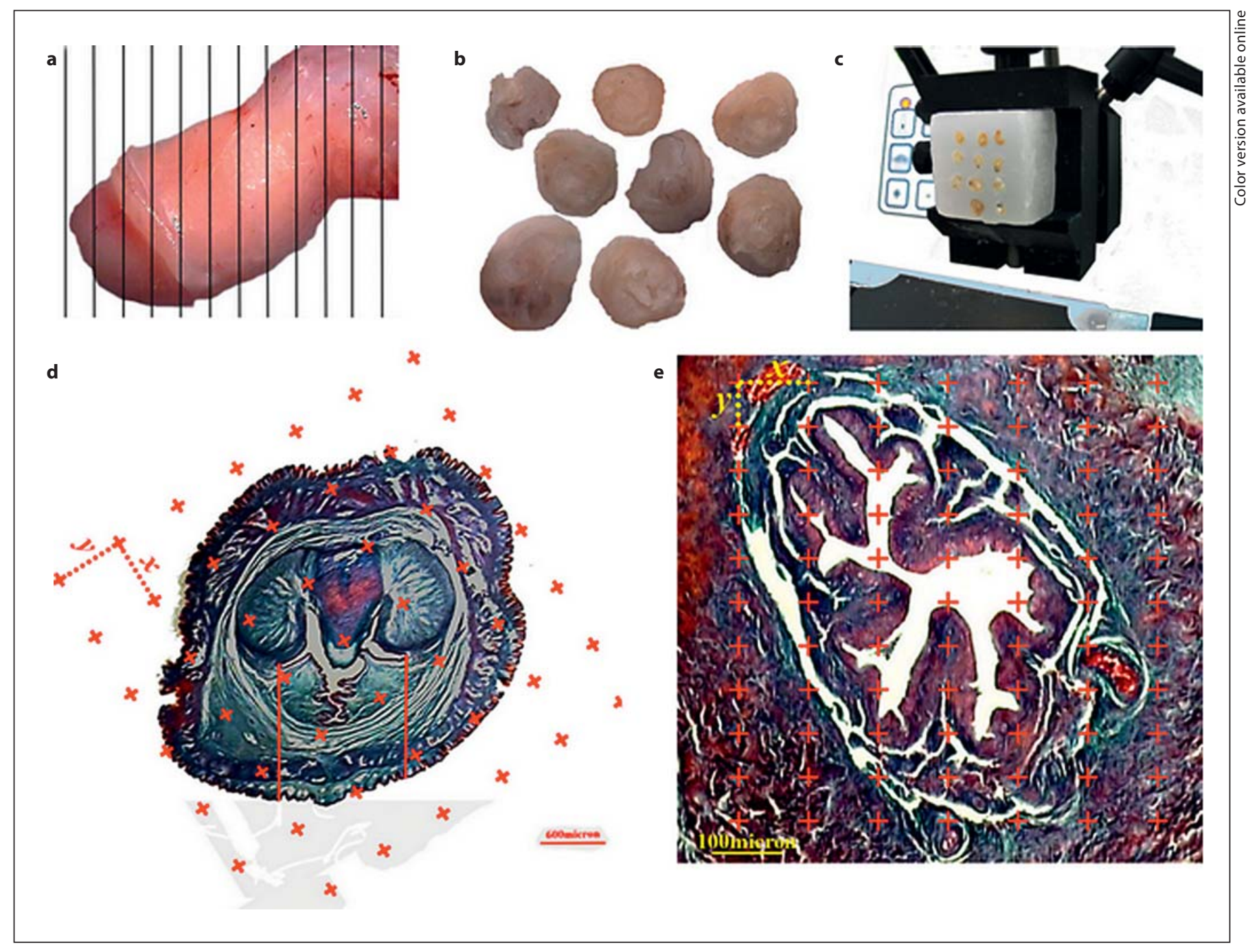

Fig. 1. Estimation of the volume of the penis body, urethral lumen and epithelium using the Cavalieri method. a The penis was removed and the length measured. The penis was sectioned into slabs with equal distances and random start. Ten to twelve transverse slabs of each penis were obtained according to penis length. b All the slabs of the penis were collected. $\mathbf{c}$ The slabs were embedded in the same block and sectioned using a microtome. $\mathbf{d}$ A test system of points was overlaid on the image of the tissue to estimate the area of each slab. e To estimate the volume of the urethral lumen and epithelium, a grid of points was superimposed on the images of the sections at a higher magnification. $x$ and $y$ were the distances between two points in two directions and were used to calculate $a(p)$.

\section{Stereological Study}

The body of each penis was cut from the base. The weight and length were measured and recorded. The volume of the penis, urethral lumen and epithelium was estimated according to the Cavalieri method [13-18] (fig. 1). The penis was sectioned into slabs with equal distances and random start. Ten to twelve transverse slabs of each penis were obtained according to penis length. The slabs of each penis were processed and embedded in the same paraffin block. Using a microtome, sections (5 and $12 \mu \mathrm{m}$ thick) were obtained and stained using Heidenhain's azan trichrome and hematoxylin and eosin. A microscopic survey was done using a video microscopy system linked to a computer and monitor. The stereological grids were generated using the software designed in the Histomorphometry and Stereology Research Centre, Shiraz, Iran. The section was analyzed using a stereomicroscope connected to a computer at a final magnification (M) of $34 \times$. A test system of points was overlaid on the image of the tissue using the stereology software. The estimation was made according to Cavalieri's principle, using the following formula:

$$
\begin{aligned}
V: & =A \cdot t \\
A: & =a(p) \cdot \Sigma P_{i}
\end{aligned}
$$




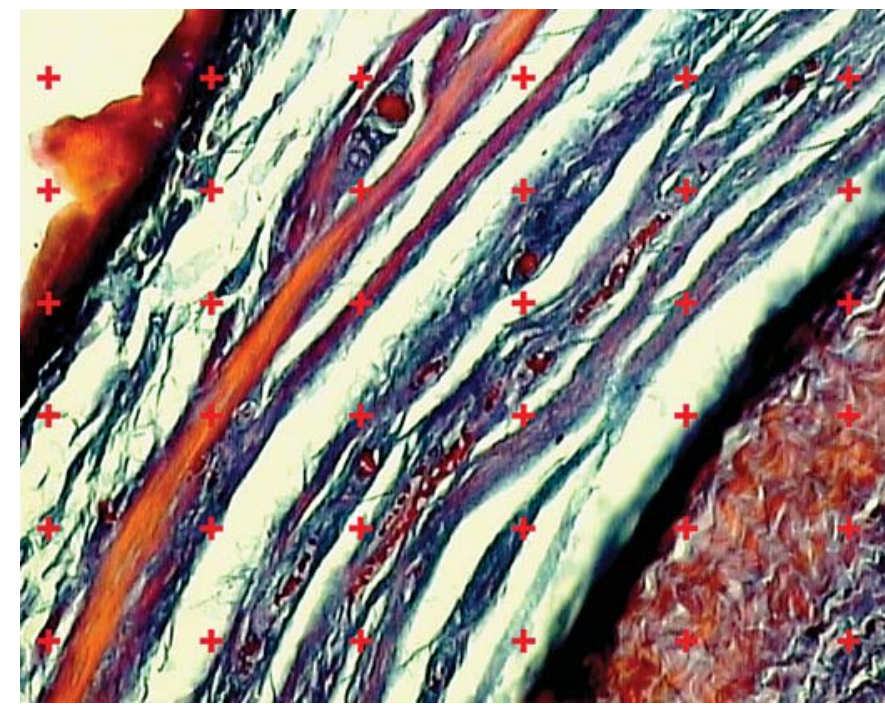

Fig. 2. Estimation of the volume density of the collagen bundles and vessels in the incised site of the penis using a point-counting method.

where $t$ was the distance between the sampled sections and the section thickness, $\Sigma P_{i}$ was the total number of points hitting the sections of the penis, and $a(p)$ was the area per point which was calculated as:

$$
a(p)=\left(\frac{\Delta X \cdot \Delta Y}{M \cdot M}\right) .
$$

To estimate the volume of the urethral lumen and epithelium, a grid of points was superimposed on the live microscopic images of the sections at a final magnification of $140 \times$. The above-mentioned method was similarly applied to obtain the volumes.

Volume density $(\mathrm{Vv})$ is the fraction of the unit volume of the tissue which is occupied by a structure [13-18]. Estimation of volume density of the collagen bundles and vessels in the incised site of the penis was done using a point-counting method (fig. 2). A grid of points was superimposed on the images of the dermis of the incised area at final magnification of $340 \times$. The density was obtained according to the formula:

$$
V_{\mathrm{v}}(\text { structure, ref }):=\mathrm{P}(\text { structure }) / \mathrm{P}(\text { ref })
$$

where $\mathrm{P}$ (structure) was the number of points hitting the collagen bundles or vessels and $\mathrm{P}(\mathrm{ref})$ was the total point number which lied on the incised area (reference area).

An optical disector method [13-18] was used to estimate the numerical density, i.e. number of the cells (lymphocytes and fibroblasts) in the unit volume of the tissue (each $\mathrm{mm}^{3}$ ) (fig. 3). This parameter was considered as a quantitative criterion for inflammation in the incised site of the penis. Optical disector means using two sections of look-up and reference. The $12-\mu \mathrm{m}$ thick sections were reviewed at a magnification of $1,400 \times$. By means of the stereology software, an unbiased counting frame was superimposed on the images of the sections viewed on the monitor. The frame avoids the 'edge effect' and biased counting of the particles, because all the nuclei profiles regardless of their shape or size were counted by the frame and had the same probability of being sampled. The optical section was moved downwards in the z-axis direction of the image. To have an unbiased counting, a guard zone was considered for estimating, i.e. the first $4 \mu \mathrm{m}$ was ignored. The look-up section was considered as the first plane appearing after the guard zone. It was achieved using a microcator (Heidenhain MT-12, Germany) that measures the z-axis traveling (height of the guard zone or disector). Any nuclear profile within the next traveling $4-\mu \mathrm{m}$ optical section (height of disector) was selected if it lay in the counting frame or touched the inclusion borders and did not touch the exclusion borders (left and lower) of the frame. The numerical density of lymphocytes and fibroblasts was estimated using the following formula:

$$
N v(\text { cells, ref }):=\frac{\sum Q^{-}}{\sum P \times h \times(a / f)} \times \frac{t}{B A}
$$

where $N v$ was numerical density, $\Sigma Q^{-}$was the total counted cells per animal, $\Sigma P$ was the total number of counted frames, $h$ was the height of the disector $(4 \mu \mathrm{m}), \mathrm{a} / \mathrm{f}$ was the area of the frame, $t$ was the real thickness of the sections estimated using a microcator on the different fields of the sections, and $B A$ was the thickness that was set for microtome advance $(12 \mu \mathrm{m})$.

\section{Statistical Analysis}

The data were compared using the Kruskal-Wallis and MannWhitney $U$ test. The results were reported as means and coefficient of variation $(\mathrm{CV}) . \mathrm{p}<0.05$ was considered statistically significant.

\section{Results}

As displayed in table 1, no significant differences were seen in weight and volume of the penis in each group. Table 2 compares the total volume of the urethral lumen and epithelium in the body of the penis. In the poliglecaprone 25 group, urethral lumen volume was higher by $\sim 56,29,31$, and $15 \%$ compared with chromic, polyglactic acid, polydioxanone, and polyglactin 910 , respectively $(p<0.001)$. In the polydioxanone group, the volume of the urethral epithelium was higher by $\sim 62$, 44,58 , and $48 \%$ compared with chromic, polyglactic acid, polyglactin 910 , and poliglecaprone 25 groups, respectively $(\mathrm{p}<0.001)$. Table 2 also compares the volume density of collagen bundles and vessels in the incised area. There were no significant differences among the five operated groups regarding volume density of collagen bundles ( $\mathrm{p}<0.001)$; however, in the poliglecaprone 25 group the volume density of the vessels was higher by $\sim 42,42,28$, and $47 \%$ compared with chromic, polyglactic acid, polydioxanone, and polyglactin 910 , respectively $(\mathrm{p}<0.001)$. 
Fig. 3. Estimation of the numerical density of lymphocytes and fibroblasts using an optical disector. a Look-up section which was the first plane to appear after the guard zone. b Any nuclear profile within the next traveling $4-\mu \mathrm{m}$ optical section (height of disector) was selected if it lay in the counting frame or touched the inclusion borders and did not touch the exclusion borders (left and lower) of the frame (here three fibroblasts).
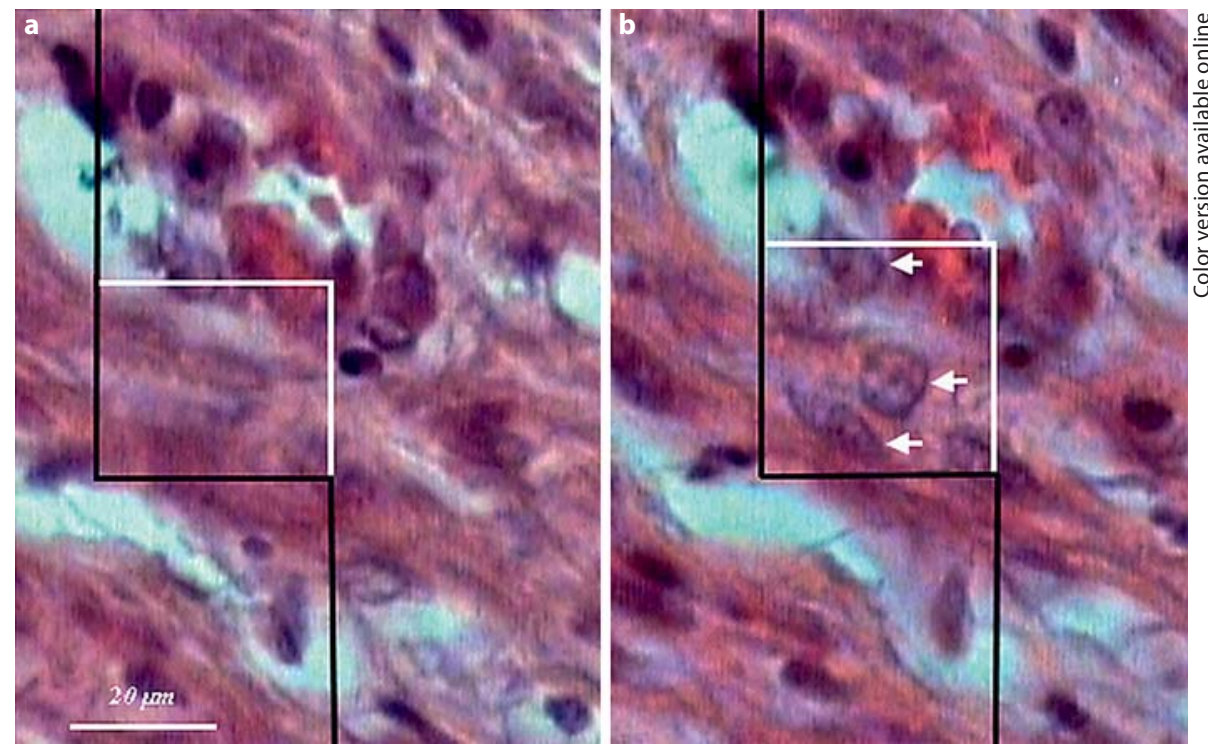

Table 1. Mean (CV) of animal weight (g), weight $(\mathrm{g})$ and volume $\left(\mathrm{mm}^{3}\right)$ of the body of the penis of rats in the six animal groups including control, chromic catgut, polyglactic acid (Vicryl), polydioxanone (PDS), polyglactin 910 (Vicryl Rapid), and poliglecaprone 25 (Monocryl) $(\mathrm{n}=5)$

\begin{tabular}{lllllll}
\hline & Control & Chromic & Polyglactic acid & Polydioxanone & Polyglactin 910 & Poliglecaprone 25 \\
\hline Animal weight & $306(0.03)$ & $308(0.04)$ & $304(0.03)$ & $308(0.03)$ & $300(0.02)$ & $306(0.05)$ \\
Penis weight & $0.31(0.03)$ & $0.32(0.06)$ & $0.30(0.03)$ & $0.31(0.03)$ & $0.29(0.03)$ & $0.31(0.03)$ \\
Penis volume & $280(0.07)$ & $280(0.07)$ & $260(0.03)$ & $270(0.03)$ & $260(0.03)$ & $270(0.03)$ \\
\hline
\end{tabular}

Table 2. Mean (CV) of the total volumes of the urethral lumen $\left(\mathrm{mm}^{3}\right)$, luminal epithelium $\left(\mathrm{mm}^{3}\right)$ in the body of the penis, volume density (\%) of the collagen bundles and vessel (\%) in the incised area of the control and operated groups including chromic catgut, polyglactic acid (Vicryl), polydioxanone (PDS), polyglactin 910 (Vicryl Rapid), and poliglecaprone 25 (Monocryl) $(\mathrm{n}=5)$

\begin{tabular}{lllllll}
\hline & Control & Chromic & Polyglactic acid & Polydioxanone & Polyglactin 910 & Poliglecaprone 25 \\
\hline Lumen & $1.8(0.20)$ & $0.45(0.66)$ & $0.73(0.27)$ & $0.71(0.3)$ & $0.87(0.45)$ & $1.03(0.23)^{*}$ \\
Epithelium & $1.6(0.12)$ & $1.1(0.45)$ & $1.6(0.18)$ & $2.9(0.75)^{* *}$ & $1.2(0.16)$ & $1.5(0.13)$ \\
Vessels & $8.1(0.13)$ & $3.6(0.29)$ & $3.6(0.19)$ & $4.5(0.31)$ & $3.3(0.10)$ & $6.3(0.25)^{*}$ \\
Collagen & $62.9(0.13)$ & $63.3(0.27)$ & $64.1(0.11)$ & $55.8(0.24)$ & $69.1(0.26)$ & $68.4(0.49)$ \\
\hline
\end{tabular}

${ }^{*} \mathrm{p}<0.001$, poliglecaprone 25 vs. (chromic, polyglactic acid, polydioxanone, and polyglactin 910).

** $\mathrm{p}<0.001$, polydioxanone vs. (chromic, polyglactic acid, polyglactin 910 and poliglecaprone 25).

Table 3. Mean (CV) of the numerical density $\left(\mathrm{n} / \mathrm{mm}^{3}\right)$ of lymphocytes and fibroblasts in the incised area of the groups including chromic catgut, polyglactic acid (Vicryl), polydioxanone (PDS), polyglactin 910 (Vicryl Rapid), and poliglecaprone $25(\mathrm{Monocryl})(\mathrm{n}=5)$

\begin{tabular}{lllllll}
\hline & Control & Chromic & Polyglactic acid & Polydioxanone & Polyglactin 910 & Poliglecaprone 25 \\
\hline Lymphocytes & $41,083(0.03)$ & $122,994(0.04)$ & $121,291(0.01)$ & $121,492(0.01)$ & $122,392(0.01)$ & $61,979(0.01)^{*}$ \\
Fibroblasts & $49,912(0.02)$ & $134,495(0.01)$ & $130,582(0.01)$ & $129,095(0.01)$ & $133,267(0.01)$ & $135,862(0.03)$ \\
\hline
\end{tabular}

${ }^{*} \mathrm{p}<0.001$, poliglecaprone 25 vs. (chromic, polyglactic acid, polydioxanone and polyglactin 910). 
Table 3 shows the comparison of the numerical density of the fibroblast and lymphocyte infiltration in the incised area. In the poliglecaprone 25 group, the lymphocyte cell count was lower by $\sim 49,48,48$, and $49 \%$ compared with the chromic, polyglactic acid, polydioxanone, and polyglactin 910 groups, respectively $(\mathrm{p}<0.001)$. There were no significant differences among the five operated groups regarding fibroblast cell count. None of the rats developed bleeding, hematoma formation or infection in the postoperation period.

\section{Discussion}

Hypospadias is a relatively common condition in pediatric urology practice affecting approximately 3.1 per 1,000 male live births [19]. Choosing appropriate suture materials for wound closure and urethroplasty depends on many factors including personal preferences, tensile strength of the suture material, the absorption time and the outcome of surgery. Although several studies have investigated this issue, data regarding the best suture material for hypospadias repair are scarce and conflicting. DiSandro and Palmer [19] demonstrated that suture materials with rapid and intermediate absorption such as polyglycolic acid and chromic provide the best results for hypospadias repair while those with delayed absorption rates such as polydioxanone are not appropriate for urethral anastomoses. Likewise, it was shown by Bartone et al. [10] that chromic is the best suture material for hypospadias repair surgery while polyglycolic acid and polydioxanone sutures should be avoided in this surgery because of their prolonged resorption and excess reaction. Contrary to these studies, Guarino et al. [6] compared different suture threads in primary hypospadias repair with polyglytone or polydioxanone. They found that the polydioxanone group had better persistence of sutures and less skin tracts at long-term followup. These results implicate that although both suture materials are adequate and appropriate for hypospadias repair in children, polydioxanone had a better cosmetic and long-term outcome because of its rapid absorption time, leading to rapid disappearance of the skin sutures and less skin tracts. As opposed to the results of DiSandro and Palmer [19] in this respect, Ulman et al. [7] also found that urethroplasty using polydioxanone suture material is associated with lower complication rate such as fistula formation. Cimador et al. [20] questioned the role of suture materials in the complication rate after flap urethroplasty procedures. They pointed out that an appropriate technique, meticulous surgery, and surgeon experience are more important factors than suture material in the flap urethroplasty procedure. Molea et al. [21] performed an experimental study comparing monofilament synthetic absorbable suture materials for skin closure in rats. They used polydioxanone, poliglecaprone 25 and glycomer 631 to close the skin in 72 rats with respect to their clinical characteristics, tissue inflammatory reaction and suture absorption times. They found that poliglecaprone 25 and glycomer 631 suture materials were less reactive than polydioxanone in the rat's skin. In another study, Yilmaz et al. [22] performed an experimental study on diabetic rats in order to compare poliglecaprone 25 , silk and catgut suture materials on wound healing. They found that although all these suture materials are well tolerated and have enough tensile strength, poliglecaprone 25 seemed to have more beneficial effects on wound healing in diabetic subjects [22].

To the best of our knowledge, this study is the first to evaluate and compare the structural and histological parameters of the penis and urethra and their changes, using stereological methods, after urethroplasty using five different absorbable suture materials. We sought to determine the best absorbable suture materials for hypospadias repair based on better collagen deposition, larger lumen volume, vascularization and less inflammation, i.e. the structural criteria of wound healing [23]. We found that using Monocryl (poliglecaprone 25) in hypospadias repair in an animal model (Sprague-Dawley rats) is associated with a maximum density of the vessel and wider urethral lumen, when compared to chromic, polydioxanone, polyglactic acid, and polyglactin 910. Poliglecaprone 25 was also associated with a lower inflammation rate as it had a minimal lymphocyte infiltration compared to the other four suture materials. This latter finding, less inflammation and lymphocyte infiltration, might be an explanation for this suture material showing a wider urethral lumen volume when compared to chromic, polyglactic acid, polyglactin 910 , and polydioxanone. It may be associated with the better outcome of urethroplasty, however further study might be required to confirm these findings. The studies should mainly be done at different times to evaluate the remodeling of the urethra.

Some limitations were noted in this study: first, it was performed on an intact urethra which has no resemblance to the urethral plate in hypospadias, and second, this is an experimental preliminary report which cannot be attributable to humans. Further clinical and biochem- 
ical studies are suggested to determine the more beneficial aspects of the suture material for urethroplasty and hypospadias surgery.

\section{Conclusion}

Chromic, polydioxanone, poliglecaprone 25, polyglactic acid, and polyglactin 910 are all suitable absorbable suture materials for urethroplasty in the rat model, however poliglecaprone 25 was found to be associated with more vessel density and a wider urethral lumen. In the polydioxanone group, more urethral epithelium was observed as compared to the other groups.

\section{Acknowledgements}

We hereby acknowledge the financial support of the ViceChancellor of Research of Shiraz University of Medical Sciences (grant No. 90-5657) for the present work which was partly extracted from a residency thesis in urology (A. Serhan) and carried out in the Histomorphometry and Stereology Research Center, Shiraz University of Medical Sciences. Ms. Elham Nadimi and Mr. Mehrdad Azadi are acknowledged for their histo-technical help.

\section{Disclosure Statement}

The authors have no conflicts of interest to disclose.

\section{References}

1 Baskin LS, Erol A, Jegatheesan P, Li Y, Liu W, Cunha GR: Urethral seam formation and hypospadias. Cell Tissue Res 2001;305:379387.

- 2 Huang WY, Chen YF, Guo YJ, Lan CF, Chang HC, Chen SC, Huang KH: Epidemiology of hypospadias and treatment trends in Taiwan: a nationwide study. J Urol 2011;185: 1449-1454.

3 Adelsberger ME, Smeak DD: Repair of extensive perineal hypospadias in a Boston terrier using tubularized incised plate urethroplasty. Can Vet J 2009;50:937-942.

-4 Hayashi Y, Kojima Y: Current concepts in hypospadias surgery. Int J Urol 2008; 15:651664.

5 Mouriquand PD, Gorduza DB, Noché ME, Targnion A: Long-term outcome of hypospadias surgery: current dilemmas. Curr Opin Urol 2011;21:465-469.

-6 Guarino N, Vallasciani SA, Marrocco G: A new suture material for hypospadias surgery: a comparative study. J Urol 2009;181 $1318-1322$.

7 Ulman I, Erikçi V, Avanoğlu A, Gökdemir A: The effect of suturing technique and material on complication rate following hypospadias repair. Eur J Pediatr Surg 1997;7:156-157.

8 Tajirian AL, Goldberg DJ: A review of sutures and other skin closure materials. J Cosmet Laser Ther 2010;12:296-302.

-9 Snodgrass W, Yucel S: Tubularized incised plate for mid shaft and proximal hypospadias repair. J Urol 2007;177:698-702.
10 Bartone F, Shore N, Newland J, King L, DuPlessis D: The best suture for hypospadias? Urology 1987;29:517-522.

11 Pillai CK, Sharma CP: Review paper: absorbable polymeric surgical sutures: chemistry, production, properties, biodegradability, and performance. J Biomater Appl 2010; 25:291-366.

12 Diegelmann RF, Evans MC: Wound healing: an overview of acute, fibrotic and delayed healing. Front Biosci 2004;9:283-289.

13 Hyde DM, Tyler NK, Plopper CG: Morphometry of the respiratory tract: avoiding the sampling, size, orientation, and reference traps. Toxicol Pathol 2007;35:41-48.

14 Gundersen HJ, Bagger P, Bendtsen TF, et al: The new stereological tools: disector, fractionator, nucleator and point sampled intercepts and their use in pathological research and diagnosis. APMIS 1988;96:857-881.

15 Gundersen HJ, Bendtsen TF, Korbo L, et al: Some new, simple and efficient stereological methods and their use in pathological research and diagnosis. APMIS 1988;96:379394.

16 Nyengaard JR: Stereologic methods and their application in kidney research. J Am Soc Nephrol 1999;10:1100-1123.

17 Abidu-Figueiredo M, Ribeiro IC, Chagas MA, Cardoso LE, Costa WS, Sampaio FJ: The penis in diabetes: structural analysis of connective tissue and smooth muscle alterations in a rabbit model. BJU Int 2011;108: 400-404.
18 Costa WS, Rebello SB, Cardoso LE, Cavalcanti AG, Sampaio FJ: Stereological and biochemical analysis of muscular and connective tissue components in the penile corpus cavernosum adjacent to the fibrous plaque of Peyronie's disease. BJU Int 2009;103:212216.

19 DiSandro M, Palmer JM: Stricture incidence related to suture material in hypospadias surgery. J Pediatr Surg 1996;31:881-884.

20 Cimador M, Castagnetti M, Milazzo M, Sergio M, De Grazia E: Suture materials: do they affect fistula and stricture rates in flap urethroplasties? Urol Int 2004;73:320-324.

- 21 Molea G, Schonauer F, Bifulco G, D’Angelo D: Comparative study on biocompatibility and absorption times of three absorbable monofilament suture materials (polydioxanone, poliglecaprone 25 , glycomer 631 ). Br J Plast Surg 2000;53:137-141.

22 Yilmaz N, Inal S, Muğlali M, Güvenç T, Baş B: Effects of poliglecaprone 25, silk and catgut suture materials on oral mucosa wound healing in diabetic rats: an evaluation of nitric oxide dynamics. Med Oral Patol Oral Cir Bucal 2010;15:e526-e530.

23 DiPietro LA: Wound healing: the role of the macrophage and other immune cells. Shock 1995;4:233-240. 